Agustina Vericat ${ }^{1}$

Alicia Bibiana Orden ${ }^{1}$

\section{El desarrollo psicomotor y sus alteraciones: entre lo normal y lo patológico}

\author{
Psychomotor development and its disorders: \\ between normal and pathological development
}

\begin{abstract}
This article discusses some aspects of psychomotor development and its disorders, with special emphasis on psychomotor retardation. Diagnostic classifications of psychomotor problems, such as DSM-IV and CIE-10, are referred to and their advantages and disadvantages are analyzed. The concept of normality as a synonym for the statistical mean in the context of psychomotor disorders is also analyzed in order to consider its dynamic and variability, thereby avoiding the normality/pathology opposition, while some issues, such as the social and cultural aspects, are highlighted, making it possible to rethink the universality and relativity of psychomotor development.
\end{abstract}

Key words Psychomotor development, Developmental retardation, Normality, Pathology
Resumen El siguiente artículo discute aspectos propios del desarrollo psicomotor (DPM) y sus alteraciones, con especial énfasis en el retraso psicomotor. Se hace referencia a las clasificaciones diagnósticas para los problemas del desarrollo como el DSMIV y el CIE 10, y se analizan sus ventajas y desventajas. También se problematiza el concepto de normalidad en tanto sinónimo de promedio estadístico en el contexto de los problemas del DPM, para considerar su dinámica y variabilidad, evitando la oposición normalidad/patología, $y$ valorando aspectos como el sociocultural que permiten repensar la universalidad y la relatividad del DPM.

Palabras clave Desarrollo psicomotor, Retraso madurativo, Normalidad, Patología
${ }^{1}$ Instituto de Desarrollo e Investigaciones Pediátricas "Prof. Dr. Fernando E. Viteri”. Hospital de Niños Sor M. Ludovica. Calle 14 Nro. 1631. 1900 La Plata Argentina. agustinavericat@gmail.com 


\section{El desarrollo psicomotor y sus características}

El término desarrollo psicomotor (DPM) se atribuye al neuropsiquiatra alemán Carl Wernicke (1848-1905), quien lo utilizó para referirse al fenómeno evolutivo de adquisición continua y progresiva de habilidades a lo largo de la infancia ${ }^{1}$. Las habilidades mencionadas comprenden la comunicación, el comportamiento y la motricidad del niño ${ }^{2}$. Illingworth ${ }^{3}$ aportó una de las definiciones más precisas expresando que el desarrollo psicomotor es un proceso gradual y continuo en el cual es posible identificar etapas o estadios de creciente nivel de complejidad, que se inicia en la concepción y culmina en la madurez, con una secuencia similar en todos los niños pero con un ritmo variable. Ambas concepciones remiten a conceptos de evolución o cambio y de gradualidad y continuidad de dichos cambios. Esto permite concebir el DPM como un proceso que posibilita al niño realizar actividades progresivamente más complejas y consecutivas o secuenciales. Por ejemplo, para adquirir la marcha, un niño debe antes poder sentarse, luego pararse y finalmente caminar.

Una de las particularidades del desarrollo psicomotor es la existencia de variaciones interindividuales. Estas variaciones en los niños pequeños, dificultan a menudo distinguir entre los cambios que podrían considerarse normales o esperables y los retrasos de maduración provenientes de desórdenes temporales o permanentes, siendo cada camino diferente y particular, con trayectorias caracterizadas por continuidades y discontinuidades ${ }^{4}$. Esto es así porque durante el desarrollo, la adquisición progresiva de funciones no se realiza según un programa secuencial rígido, sino que por el contrario, presenta variaciones en el ritmo o tiempo de desarrollo que requiere cada nueva habilidad lograda, así como también en la edad cronológica en que es esperable el logro de una habilidad o evento determinado. Un ejemplo conocido es la adquisición de la marcha, que puede variar entre los 9 y los 16 meses de edad del niño ${ }^{5}$.

El desarrollo psicomotor (DPM) resulta de la interacción de factores propios del individuo (biológicos) y aquellos vinculados a determinantes del contexto psicosocial (familia, condiciones de vida, redes de apoyo, entre otras). Según Young y Fujimoto Gómez 6 el desarrollo humano es moldeado por una interacción dinámica y continua entre la biología y la experiencia. La cultura influye en cada uno de los aspectos del desarrollo y esto se ve reflejado en las prácticas de crianza y creen- cias, diseñadas para promover una saludable adaptación. Durante su maduración, los niños no son sujetos pasivos, puramente receptores de información o enseñanzas, sino que participan activamente en este proceso, explorando y dominando gradualmente el ambiente que los rodea. Las relaciones sociales del entorno del niño son básicas para un desarrollo saludable, generando factores de protección o resiliencia ${ }^{7}$; pero también pueden constituir factores de riesgo ${ }^{8}$.

\section{Alteraciones o problemas del desarrollo}

Cuando se hace referencia a desarrollo psicomotor normal se habla de un proceso que permite al niño adquirir habilidades adecuadas para su edad. No obstante, como se mencionó, existe gran variabilidad en la edad en la adquisición o alcance de diferentes habilidades. Esto es relevante porque da cuenta de la dificultad de establecer claramente un límite entre lo "normal" y lo "patológico" $"$. En general, ambas esferas son diferenciadas con criterios de normalidad estadística bajo los términos desvío, significación y promedio. Así Poó Argüelles ${ }^{5}$ planteó que lo patológico es apartarse de una manera significativa de lo esperado para la edad, en un área concreta o en la globalidad e Illingworth sostuvo lo único que se puede decir es que cuanto más lejos del promedio se encuentre un niño, en cualquier aspecto, es menos probable que sea normal ${ }^{5}$. En esta perspectiva, cuando el DPM presenta características peculiares o diferentes a la "norma", se está en presencia de alteraciones o problemas del desarrollo. ¿Pero cuán apartado de la norma debe estar el DPM para ser considerado patológico? En general es sencillo estar de acuerdo en lo "muy patológico", pero no tanto cuando se intentan definir ciertas alteraciones o trastornos, que pueden discurrir entre ambos extremos.

El DPM puede presentar variantes o alteraciones diversas. El retraso psicomotor, los diferentes tipos de trastornos del desarrollo y los problemas inaparentes del desarrollo son ejemplos de este tipo de alteraciones. El retraso psicomotor es uno de los cuadros más frecuentemente detectados en niños pequeños. Narbona y Schlumberger ${ }^{10}$ lo definieron como un diagnóstico provisional, en donde los logros del desarrollo de un determinado niño durante sus primeros tres años de vida aparecen con una secuencia lenta para su edad y/o cualitativamente alterada. El término retraso psicomotor, entonces, se suele mantener hasta que pueda establecerse un diagnóstico definitivo a través de pruebas formales ${ }^{11}$. Alvarez Gómez et 
al. ${ }^{12}$ sostienen que, debido a que es un término muy indefinido, no debería utilizarse más allá de los tres a cinco años de edad del niño, cuando ya se pueden realizar tests que miden la capacidad intelectual.

En España el término retraso psicomotor se utiliza como sinónimo de retraso del desarro$1 \mathrm{lo}^{10}$, mientras que en América Latina es más frecuente el término retraso madurativo ${ }^{13}$. Álvarez Gómez et al, por otra parte, definen al retraso del desarrollo como una demora o lentitud en la secuencia normal de adquisición de los hitos del desarrollo, por lo cual para estos autores no existe nada intrínsecamente anormal, los hitos madurativos se cumplen en el orden esperado, sólo que en forma más lenta ${ }^{12}$. Esto implica que, a largo plazo, el niño adquirirá las habilidades deficitarias y siempre seguirá un orden específico en la adquisición de las mismas.

Por lo anteriormente mencionado, el niño con retrasos en su desarrollo puede normalizarse a largo plazo y, cuando esto no ocurre, será diagnosticado con una cierta patología. Narbona y Schlumberger ${ }^{10}$ contemplaron las diferentes posibilidades diagnósticas en las que puede desembocar un cuadro que inicialmente se manifestó como un retraso psicomotor de la siguiente manera: puede ocurrir que el retraso sea una $\mathrm{va}$ riante normal del desarrollo, en cuyo caso se normalizará espontáneamente antes de la edad preescolar. Puede que en realidad sea un verdadero retraso, debido a déficit en la estimulación por parte del entorno familiar y social, que podría ser normalizado si se adecuara la educación y el ambiente del niño (retraso de etiología ambiental); o bien deberse a enfermedad crónica extraneurológica (cardiopatía congénita, enfermedad respiratoria, desnutrición, entre otras), compensándose en la medida en que mejora la enfermedad general de base. Por otra parte, un retraso puede deberse al efecto de un déficit sensorial aislado, como la sordera neurosensorial congénita o ser la primera manifestación de una futura deficiencia mental, cuyo diagnóstico definitivo en los casos leves, no suele evidenciarse hasta el final de la edad preescolar. Otra posibilidad es que sea la primera manifestación de una encefalopatía crónica no evolutiva, un trastorno neuromuscular congénito de escasa o nula evolutividad, la primera manifestación de una futura torpeza selectiva en la psicomotricidad fina y/o gruesa (trastorno del desarrollo de la coordinación, frecuentemente asociado a la forma disatencional del TDAH), o el inicio de un trastorno global del desarrollo (trastorno de tipo autista) ${ }^{10}$.
A veces es relativamente sencillo percibir si el retraso puede ser transitorio o no. En los casos en que los retrasos están asociados a otros signos o características físicas o dismorfias, por ejemplo, es más frecuente que se trate de un cuadro que tienda a mantenerse en el tiempo. Lo mismo ocurre en el retraso global del desarrollo donde hay alteración de dos o más áreas o campos del desarrollo, manifestándose un retraso significativo, correspondiente a dos o más desviaciones estándar inferior a la media en pruebas acorde a la edad del niño ${ }^{14}$. Algunos ejemplos de trastornos globales del desarrollo son el autismo, el síndrome de Asperger o el síndrome de Rett. Cuando el problema del desarrollo es leve o sutil, puede no ser fácilmente evidenciable y para detectarlo es necesario realizar una prueba de pesquisa o screening ${ }^{15,16}$. En estos casos podría hablarse de trastornos inaparentes del desarrollo psicomotor. Dado que la mayoría de los lactantes y preescolares con dificultades del desarrollo no tienen signos obvios de enfermedad, por lo menos en un inicio, ni factores de riesgo que lo sugieran, la identificación de estos niños aparentemente sanos suele constituir un verdadero desafío ${ }^{1}$. Los trastornos inaparentes del desarrollo plantean tal vez la discusión más difícil en esta área y transcurren en un límite difuso entre lo "normal y patológico".

\section{Clasificaciones diagnósticas de los problemas del DPM}

Una de las dificultades halladas al incursionar en los problemas del desarrollo es su clasificación. Existen numerosas clasificaciones diagnósticas, entre ellas dos sistemas principales o de uso más difundido. Una es la establecida por la Asociación Norteamericana de Psiquiatría, el Manual Diagnóstico y Estadístico de Trastornos Mentales en su cuarta revisión (DSM IV), que constituye el sistema más utilizado para la investigación internacional. La otra es la Clasificación Internacional de Enfermedades (CIE 10) desarrollada por la Organización Mundial de la Salud. Esta última incorpora a todos los problemas del desarrollo bajo la denominación de Trastornos del desarrollo psicológico. Habitualmente se los denomina simplemente trastornos del desarrollo y son un amplio grupo de problemas de inicio en la primera o segunda infancia, de curso estable, que varían desde dificultades escolares hasta problemas psicomotores. Una de las características principales es que presentan deterioro o retraso del desarrollo de las funciones rela- 
cionadas con la maduración biológica del sistema nervioso central. En la mayoría de los casos, las funciones afectadas son el lenguaje, las funciones viso-espaciales y la coordinación de los movimientos. En el Cuadro 1 se listan los trastornos incluidos por la CIE 10.

Otras clasificaciones diagnósticas para los problemas del desarrollo coinciden parcialmente con la CIE 10. Una de las más aceptadas en salud mental es el Manual de Diagnóstico y Estadística de las Enfermedades Mentales (DSM IV) de la Asociación Americana de Psiquiatría ${ }^{17}$. Esta clasificación (Cuadro 2) incorpora a los trastornos del desarrollo infantil bajo la categoría Trastornos de inicio en la infancia, la niñez o la adolescencia incluyendo en este grupo al retraso mental, a los trastornos del aprendizaje, a la de las habilidades motoras, a los de la comunicación, a los trastornos generalizados del desarrollo, a los trastornos por déficit de atención y comportamiento perturbador, entre otros ${ }^{18}$.

Una ventaja de este tipo de clasificaciones es que posibilitan organizar los cuadros clínicos y otorgan a los profesionales un lenguaje en común. Sin embargo, muchas veces imponen rótulos a los pacientes, con lo cual se pueden limitar las expectativas que se tiene sobre el niño ${ }^{19}$. La infancia es un período de cambios continuos por lo que a veces es difícil encajar un trastorno en una estructura clasificatoria rígida; es por eso que a las mencionadas clasificaciones se les objeta que, tanto el factor evolutivo como las diferencias de expresión de los síntomas en relación a la edad estén poco considerados en los apartados dedicados a los niños. A su vez, las secciones referidas exclusivamente a los niños son muy reducidas,

Cuadro 1. Clasificación de los trastornos del desarrollo de la CIE 10

\begin{tabular}{|l|}
\hline Trastornos del desarrollo psicológico (F80-89) \\
\hline $\begin{array}{l}\text { Trastornos específicos del lenguaje y del habla } \\
\text { (F80) }\end{array}$ \\
\hline $\begin{array}{l}\text { Trastornos del desarrollo específicos del } \\
\text { aprendizaje escolar (F81) }\end{array}$ \\
\hline $\begin{array}{l}\text { Trastornos del desarrollo específicos del desarrollo } \\
\text { psicomotor (F82) }\end{array}$ \\
\hline Trastornos del desarrollo específicos mixtos (F83) \\
\hline Trastorno generalizado del desarrollo (F84) \\
\hline Otros trastornos del desarrollo psicológico (F88) \\
\hline
\end{tabular}

Fuente: OPS 20 como en el caso de la DSM IV, que dedica un solo capítulo a los problemas de inicio en la infan$\mathrm{cia}^{17}$. Por último, aunque los sistemas de clasificaciones diagnósticas van delimitando un lenguaje común en sus sucesivas versiones, los resultados son poco satisfactorios en lo que respecta a niños $^{21}$.

\section{Relativizando lo normal y lo patológico}

Puede decirse que, universalmente, el desarrollo psicomotor es un proceso evolutivo y continuo, que sigue una secuencia similar en todos los niños. Sin embargo, establecer qué es lo normal en el desarrollo psicomotor es dificultoso, porque el proceso de maduración psicomotriz no se realiza de manera rígida ni estereotipada ${ }^{2}$. Esto plantea algunas preguntas ¿Qué es normal? ¿Lo "normal" es "normal" para todos? Rosato et al. ${ }^{22}$ introdujeron el concepto de "ideología de la normalidad" para analizar los procesos de producción de discapacidad. Esta ideología opera sustentada en una lógica binaria, donde "anormalidad" no describe una cosa singular ni estable sino que más bien es referida a los límites que marca el termino opuesto, la normalidad.

Illingworth, afirmaba que los retrasos del desarrollo psicomotor son pensados en función de alteraciones en la secuencia del desarrollo y en la adquisición de ciertas pautas que se demoran

Cuadro 2. Trastornos de inicio en la infancia niñez o adolescencia según el DSM IV.

\begin{tabular}{|l|}
\hline \multicolumn{1}{|c|}{$\begin{array}{c}\text { Trastornos de inicio en la infancia, } \\
\text { niñez o adolescencia }\end{array}$} \\
\hline Retraso mental \\
\hline Trastornos del aprendizaje \\
\hline Trastornos de las habilidades motoras \\
\hline Trastornos de la comunicación \\
\hline Trastornos generalizados del desarrollo \\
\hline $\begin{array}{l}\text { Trastornos por déficit de atención y } \\
\text { comportamiento perturbador }\end{array}$ \\
\hline $\begin{array}{l}\text { Trastornos de la ingestión y de la conducta } \\
\text { alimentaria de la infancia o de la niñez }\end{array}$ \\
\hline Trastornos de tics \\
\hline Trastornos de la eliminación \\
\hline $\begin{array}{l}\text { Otros trastornos de la infancia, la niñez o la } \\
\text { adolescencia }\end{array}$ \\
\hline
\end{tabular}

Fuente: APA, 2000 (19) 
más que la "norma". Desde una perspectiva estadística, cuanto más lejos se encuentre un niño del promedio, es menos probable que sea normal ${ }^{23}$. Canguilhem ${ }^{24}$ debatió con aquellos que identificaban norma con promedio y sostenían que los valores considerados promedios estadísticos otorgarían las medidas ciertas de lo que debía ser considerado normal para un organismo. Si la norma es más que el promedio algunos niños ubicados en los extremos de una campana de Gauss, podrían pertenecer a la norma (falsos positivos), en vez de ser clasificados como "anormales”. En su obra Lo Normal y lo Patológico Canguilhem ${ }^{24}$ afirmaba que lo normal es fijado por convención. Consideraba que los promedios (constantes) fisiológicos expresan normas colectivas de vida histórica y socialmente cambiantes. Sostenía que no es el promedio el que establece lo normal, sino que por el contrario las constantes fisiológicas expresan normas de vida que no son el resultado de hábitos individuales sino de valores sociales y biológicos. Para este autor la variación era parte de la normalidad "siendo que lo normal no tiene la rigidez de una determinante que valga para toda la especie, sino la flexibilidad de una norma que se transforma en relación a las condiciones individuales, entonces es claro que el límite entre lo normal y lo patológico se hace impreciso"25. Siguiendo a Caponi ${ }^{25}$ esto implica asumir la construcción subjetiva de formas de vida y modos de ser fisiológicos y que es a través de la variación de las normas sociales y vitales que se producen variaciones en los promedios estadísticos que consideramos constantes funcionales. La perspectiva de Canguilhem hace repensar la falsa oposición normal/ patológico en tanto lo normal comprende lo variable. Por otra parte permite relativizar la normalidad no sólo en función de valores biológicos sino sociales. Esto posibilita rescatar la visión dinámica del DPM, considerando al retraso psicomotor como una entidad provisoria, que se produce en un continuum (más que en una oposición) normalidad-patología.

Una segunda cuestión, abordada especialmente por la psicología y la antropología, es la relacionada a la universalidad y la relatividad del desarrollo. El universalismo y el relativismo son vistos como dos emergentes de la relación entre cultura y psicopatología, y remiten a las nociones de etic y emic, es decir lo universal y lo particular del comportamiento humano ${ }^{26}$. El primer enfoque permite construir categorías aplicables a todos los grupos humanos, dando sustento a las clasificaciones del tipo CIE 10 y DSM IV ${ }^{27}$. El enfoque emic, por el contrario, desacuerda con las categorías diagnósticas generalizables, proponiendo el conocimiento de los significados específicos que cada cultura otorga a un determinado síntoma o dolencia $^{28}$. En este punto es necesario pensar el desarrollo normal en función de la percepción (subjetiva) y del contexto cultural desde donde se identifican/definen y adquieren significado los eventos del desarrollo infantil. Lev Vygotsky ${ }^{29}$ afirmaba que el niño es un ser social desde que nace y que en la interacción con los demás va a residir la clave de su desarrollo. Para la psicología cultural y transcultural las prácticas sociales promueven comportamientos culturalmente específicos. Así, la diversidad del comportamiento es entendida como un producto de las relaciones entre el contexto cultural y el desarrollo humano ${ }^{30}$. De tal modo, algunos contextos pueden favorecer el desarrollo de un área madurativa en particular. Por ejemplo, entre los Mbyá-Guaraní, el ser considerado como un Mbyá requiere dos atributos: que el niño hable y camine erguido, por lo que se favorecen estos aspectos en la crianza ${ }^{31}$. También en los Mapuche, la adquisición temprana del lenguaje es un atributo deseable en un niño, por lo que es una de las actividades que se favorecen en primera instancia ${ }^{32}$. Este componente subjetivo en la percepción de conductas como normales o anormales, es el que vincula la salud mental (normalidad) a la condición de "adaptado" o "no adaptado" a las exigencias de la cultura del grupo de pertenencia $^{31}$. Al respecto, el antropólogo Meyer Fortes, citado por Nunes ${ }^{33}$, señaló que no son las fases de maduración biológica, sino las relaciones que el individuo establece dentro de su grupo doméstico y de la sociedad como un todo, las que delinean y expresan los cambios dentro del ciclo de vida.

El abordaje del desarrollo infantil y sus variantes, debe reconocer la diversidad del comportamiento humano, pensada en términos de particularidades culturales (generalmente asociadas a pertenencias étnicas específicas), pero como sostienen Colángelo et al. ${ }^{34}$ debe evitarse un culturalismo ingenuo, que llevaría por ejemplo a concebir las poblaciones indígenas como comunidades homogéneas, regidas por pautas tradicionales atemporales y excluye otras dimensiones presentes en las sociedades complejas, tales como la desigualdad social. Según estas autoras la infancia es una construcción donde se conjugan estas dimensiones, entre otras; señalando la complejidad y variedad de las relaciones que pueden establecerse entre los procesos de crecimiento y desarrollo y la edad social ${ }^{34}$. En este sentido 
señalan que la definición de "la" niñez, qué es adecuado para cada edad y qué comportamientos o características - por no adecuarse a lo establecido- caen en el terreno de lo anormal, no es un dato de la naturaleza, sino el resultado de una disputa por imponer una visión particular como la legítima y universal.

\section{Breves reflexiones}

Las consideraciones hechas con anterioridad ponen de manifiesto que la dificultad hallada en la categorización de lo normal/patológico, tam- bién rige en lo inherente a los instrumentos utilizados para su diagnóstico y/o pesquisa cuyo resultado informa si un niño "aprobó" o "no aprobó", en base a un punto de corte. Estos puntos de corte dejan lejanas las consideraciones del entorno sociocultural y ambiental del niño donde ciertas pautas son más estimuladas y valoradas que otras. Un abordaje reflexivo del desarrollo infantil deberá incluir una mirada más relativista del comportamiento humano, evitando la interpretación de los problemas del DPM desde una visión dicotómica para contemplarlos como un espectro de síntomas variables, dinámicos y modificables en el tiempo.

\section{Colaboradores}

A Vericat fue responsable por la investigación bibliográfica, discusión de textos y redacción del manuscrito. AB Orden trabajo en la discusión de textos, redacción y organización general del manuscrito. 


\section{Referencias}

1. Schonhaut L, Álvarez J, Salinas P. El pediatra y la evaluación del desarrollo psicomotor. Rev Chil Pediatr 2008; 79(Supl. 1):26-31.

2. Poó Arguelles P. Desarrollo psicomotor. La normalidad y los signos de alerta. Sociedad Española de Pediatría Extrahospitalaria y Atención Primaria 2008, 53-55. [sítio en Internet]. [acesado en 2013 ago 4]. Disponible en: http: //www.sepeap.org/imagenes/ secciones/Image/_USER_/MR_Psicomotor_ normalidad_signos_alerta.pdf.

3. Illingworth RS. El Desarrollo Infantil en sus Primeras Etapas. Barcelona: Editorial Médica y Técnica S.A.; 1983.

4. Young ME. Desarrollo del Niño en la Primera Infancia: Una Inversión en el Futuro. Washington: $\mathrm{Pu}$ blicaciones Banco Mundial; 2003.

5. Póo Arguelles P. Desarrollo psicomotor: características evolutivas de 0-3 años, signos de alerta. Resúmenes de la V Reunión Anual de la Sociedad Asturiana de Pediatría de Atención Primaria. Oviedo: Sociedad Asturiana de Pediatría de Atención Primaria; 2006.

6. Young ME, Fujimoto Gómez G. Desarrollo infantil temprano: lecciones de los programas no formales. Acción pedagógica 2004; 13(2):186-198.

7. Cyrulnik B. Los Patitos Feos. La resiliencia. Una Infancia infeliz no determina la vida. Madrid: Gedesa; 2005 .

8. Landers C, Mercer R, Molina H, Young ME. Desarrollo Integral en la Infancia: Una prioridad para la Salud. Santiago de Chile: OPS; 2006.

9. Fernández Álvarez E, Póo Argüelles P. Desarrollo psicomotor. En: Fejerman N, Fernández Álvarez E. Neurología Pediátrica. Buenos Aires: Editorial Médica Panamericana; 2007.

10. Narbona García J, Schlumberger E. Retraso Psicomotor. En: Narbona García J, Casas Fernández C, coordinadores. Protocolos de Neurología Pediátrica. Madrid: SENP-AEP; 2008.

11. Accardo PJ, Whitman BY. Dictionary of Developmental Disabilities Terminology. London: Paul Brookes; 2001.

12. Álvarez Gómez M, Soria Aznar J, Galbe SánchezVentura J. Importancia de la vigilancia del desarrollo psicomotor por el pediatra de atención primaria: revisión del tema y experiencia de seguimiento en una consulta en Navarra. Rev Pediatr Aten Primaria 2009; 11(41):65-78.

13. Contreras MM. Clasificaciones diagnósticas de los trastornos del desarrollo. En: Lejarraga $\mathrm{H}$, editor. Desarrollo del Niño en Contexto. Buenos Aires: Paidós; 2004. p. 143-176.

14. Shevell M. Retraso global del desarrollo y retraso mental o discapacidad intelectual: concepto, evaluación y etiología. Pediatr Clin N Am 2008; 55(5):1071-1084.

15. Salamanco G, D'Anna C, Lejarraga H. Tiempo requerido para la administración de una prueba de pesquisa de trastornos del desarrollo psicomotor infantil. Arch. argent. pediatr 2004; 102(3):165-169.
16. Vericat A, Orden AB. Herramientas de screening del desarrollo psicomotor en Latinoamérica. Rev chil pediatr 2010; 81(5):391-401.

17. Raheb C. Clasificaciones en Paidopsiquiatría. Barcelona: Universidad Autónoma de Barcelona; 2007.

18. American Psychiatric Association. Diagnostical and Statical Manual of Mental Disorders. $4^{\text {th }}$ edition. Washington: DSM IV TR; 2000

19. Lejarraga H. Enfoque pediátrico del desarrollo y sus problemas. En: Lejarraga H, editor. Desarrollo del Niño en Contexto. Buenos Aires: Paidós; 2004. p. 341-378.

20. Organización Panamericana de la Salud (OPAS). Clasificación Estadística Internacional de Enfermedades y Problemas Relacionados con la Salud. Décima revisión. (CIE-10) Manual de instrucciones. Vol. 1. Washington: OPAS; 2000.

21. Lasa Zulueta A. Hiperactividad y trastornos de la personalidad II: Sobre la personalidad límite. Cuadernos de psiquiatría y psicoterapia del niño y del adolescente 2003; 34-35:5-117.

22. Rosato A, Angelino A, Almeida ME, Angelino C, Kippen E, Sánchez C, Spadillero A, Vallejos I, Zuttión B, Priolo M. El papel de la ideología de la normalidad en la producción de discapacidad. Ciencia, Docencia y Tecnología 2009; 20(39):87-105.

23. Illingworth RS. El Desarrollo del Lactante y el Niño. Novena edición. Madrid: Ed. Churchill Livingstone; 1992.

24. Canguilhem G. Lo normal y lo patológico. Buenos Aires: Siglo Veintiuno Editores; 1971.

25. Caponi S. Georges Canguilhem y el estatuto epistemológico del concepto de salud. Hist. ciênc. saúdeManguinhos 1997; 4(2):287-307.

26. Berry J, Poortinga Y, Segal M, Dasen P. Cross-Cultural Psychology. Research and applications. New York: Cambridge University Press; 1992

27. Contini de González EN. Multiculturalismo y psicopatología: perspectivas en evaluación psicológica. Psicodebate 2006; 3:91-106.

28. Kleinman A. Patients and healers in the context of culture: An exploration of the borderland between anthropology, medicine, and psychiatry. Berkeley: University of California Press; 1981.

29. Vygotsky L. Mind in society. Cambridge: Harvard University Press; 1978.

30. Berry J. Preface. En: Berry J, Segall MH, Kagitçibasi, editors. Handbook of Cross-Cultural Psychology. Boston: Allyn and Bacon; 2000.

31. Remorini C. Aporte a la Caracterización Etnográfica de los Procesos de Salud-Enfermedad en las Primeras Etapas del Ciclo Vital en Comunidades Mbyá-Guaraní de Misiones, República Argentina [tesis]. La Plata: Editorial de la UNLP; 2008.

32. Arias S. Enseñanza aprendizaje del niño mapuche en el ámbito familiar [tesis]. Temuco: Universidad Católica de Temuco; 2001. 
33. Nunes A. A sociedade das crianças A'uwe-xavante. Por uma antropologia da criança. Temas de Investigação No 8. Lisboa: Instituto de Inovação Educacional, Ministério da Educação de Portugal; 1999.

34. Colángelo MA. La mirada antropológica sobre la infancia. Reflexiones y perspectivas de abordaje. Mesa: Infancias y juventudes. Pedagogía y formación. Seminario Internacional La formación docente entre el siglo XIX. Buenos Aires: Mimeo; 2003.

Artigo apresentado em 15/02/2012

Aprovado em 15/03/2012

Versão final apresentada em 23/03/2012 